\title{
Highly Fluorescent Silicon Nanocrystals Stabilized in Water
}

\section{using Quatsomes}

Dorothy A. Silbaugh ${ }^{\dagger, \S}$, Lidia Ferrer-Tasies ${ }^{\ddagger, \perp,}$, Jordi Faraudo ${ }^{\ddagger}$, Jaume Veciana ${ }^{\ddagger, \perp}$, Nora Ventosa $^{\ddagger, \perp *}$, Brian A. Korgel ${ }^{\dagger *}$

${ }^{\dagger}$ McKetta Department of Chemical Engineering and Texas Materials Institute, The University of Texas at Austin, Austin, TX, 78712-1062, United States.

${ }^{\ddagger}$ Institut de Ciència de Materials de Barcelona (ICMAB-CSIC), Esfera UAB; Campus UAB s/n; E-08193 Cerdanyola del Vallès, Spain.

${ }^{\perp}$ CIBER de Bioingeniería, Biomateriales y Nanomedicina (CIBER-BBN).

$\S$ These authors contributed equally to this work.

*Corresponding authors: korgel@che.utexas.edu; ventosa@icmab.es

\begin{abstract}
Fluorescent silicon (Si) nanocrystals (2.8 nm diameter) were incorporated into surfactant assemblies of cetyl trimethylammonium bromide (CTAB) and cholesterol, called quatsomes. In water, the quatsome-Si nanocrystal assemblies remain fluorescent and well-dispersed for weeks. In contrast to Si nanocrystals, alkanethiol-capped gold (Au) nanocrystals do not form stable dispersions in water with quatsomes. Cryogenic transmission electron microscopy (cryo-TEM) confirmed that the Si nanocrystal-quatsome structures do not change over the course of several weeks. The long-term stability of the Si nanocrystal-quatsome assemblies, their fluorescence and biocompatibility makes them attractive candidates for medical applications.
\end{abstract}




\section{INTRODUCTION}

Semiconductor nanocrystals, or quantum dots, have been used extensively to study biological systems due to their fluorescence properties and photostability. ${ }^{1-5}$ Silicon nanoparticles offer biocompatibility and photoluminescence that is well-suited for medical imaging using near-infrared (NIR) or long-wavelength visible light. ${ }^{3,6-8}$ The long fluorescence lifetimes of Si nanocrystals also make them well-suited for multispectral time-gated imaging applications. ${ }^{9}$ For these applications, Si nanocrystals must be dispersed in aqueous media and retain their fluorescence. This has been accomplished by capping Si nanocrystals with organic ligands bearing polar functional groups or by coating hydrophobic nanocrystals with surfactant or amphiphilic polymer. ${ }^{3,7,8,10,11}$ By incorporating nanocrystals into surfactant assemblies, such as micelles or vesicles, additional functionality might be achieved. ${ }^{1,711-18}$ For example, liposomal vesicles are especially interesting since they are medically-accepted pharmaceutical carriers and could provide a therapeutic delivery vehicle combined with the imaging capabilities of the nanocrystals. ${ }^{19-21}$ However, liposomes can suffer from aggregation and non-uniformity, which can negatively impact pharmacological properties and pharmaceutical properties.

Here, we show that non-liposomal vesicular structures, called quatsomes, can be highly effective dispersing agents and carriers for Si nanocrystals, with long-term dispersion stability and fluorescence. Quatsomes are composed of sterols and quaternary ammonium surfactants that self-assemble into vesicular bilayers. ${ }^{22}$ They are extremely stable and have been shown to remain dispersed for several years with high uniformity in size and lamellarity. ${ }^{23-25}$ Quatsomes have antibacterial and anti-biofilm properties, and have been used successfully to prepare stable multifunctional vesicle-biomolecule conjugates, to incorporate fluorene-based probes that are not soluble in water, and to protect ESIPT fluorophores in aqueous media. ${ }^{24,26-28}$ Furthermore, 
quatsomes are largely unaffected by changes in temperature or dilution, making them ideal candidates for use in vivo. ${ }^{22,25}$ Membrane components of quatsomes are available at pharmaceutical grade. Quaternary ammonium surfactants are well-known antiseptics and have a long history of use in a wide range of both human and veterinary products. In the present paper, quatsomes composed of cholesterol and cetyl trimethyl ammonium bromide (CTAB) were been used as a quatsome model, but other quatsome-like structures can be prepared using different quaternary ammonium surfactants, such as myristalkonium chloride (MKC) ${ }^{25,28} \mathrm{CTAB}$ is widely used in topical medicinal formulations, but MKC (a C14 homolog of benzalconium chloride) has been extensively used as an antimicrobial preservative in many ophthalmic and parenteral formulations (see FDA inactive ingredients database). ${ }^{29}$ As a proof of concept, Si nanocrystals with orange fluorescence (2.8 $\mathrm{nm}$ diameter, photoluminescence quantum yields of about $15 \%$ ) were integrated into quatsomes composed of cholesterol (chol) and cetyl trimethyl ammonium bromide (CTAB) to form stable, fluorescent nanocrystal dispersions in aqueous media. In contrast, dodecanethiol-capped gold $(\mathrm{Au})$ nanocrystals with similar size (1.8 $\mathrm{nm}$ diameter) did not associate with similar quatsome assemblies.

\section{EXPERIMENTAL SECTION}

Materials. Hydrogen silsesquioxane (HSQ) was purchased from Dow Corning. Hydrofluoric acid (HF, 48\%) was purchased from Macron Fine Chemicals. Ethanol was purchased from Pharmco-Aaper. CTAB (high purity grade) was purchased from Amresco. Chol was purchased from Anatrace. 1-Dodecanethiol (98\%) was purchased from Acros Organics. 1octene (98\%), gold(III) chloride trihydrate ( $\geq 99.9 \%)$, tetraoctylammonium bromide (TOAB, $98 \%$ ), and sodium borohydride ( $\geq 98.0 \%)$ were purchased from Sigma-Aldrich. Hydrochloric 
Acid (HCl), hexanes ( $\geq 98.5 \%$ ), chloroform $(\geq 99.8 \%)$, and toluene $(\geq 99.5 \%)$ were purchased from Fisher. 1,2-dioleoyl-sn-glycero-3-phosophocholine (DOPC) and 1,2-dioleoyl-sn-glycero-3(1'-rac-glycerol) (DOPG) in chloroform were purchased from Avanti Polar Lipids. Carbon dioxide was obtained from Matheson and nitrogen was obtained from the University of Texas Department of Physics Cryolab. Water used in all experiments was high purity water (18.2 $\mathrm{M} \Omega / \mathrm{cm}$ ) and was obtained from a Millipore Synergy Ultrapure water system.

Silicon Nanocrystal Synthesis. Silicon nanocrystals were synthesized following procedures reported in Hessel, et al. ${ }^{8} 40 \mathrm{~mL}$ of HSQ was degassed and then heated to $1100^{\circ} \mathrm{C}$ for 60 min in a tube furnace under forming gas $\left(90 \% \mathrm{~N}_{2} / 10 \% \mathrm{H}_{2}\right)$ flow. The resulting brown reaction product was then ground with a mortar and pestle, followed by further size reduction by mechanical shaking in a wrist action shaker with borosilicate beads for 9 hr. The final brown powder consists of $\mathrm{Si}$ nanocrystals embedded in an $\mathrm{SiO}_{2}$ matrix. To liberate the nanocrystals from the matrix, $0.6 \mathrm{~g}$ of powder was etched in the dark with $2 \mathrm{~mL} \mathrm{HCl}$ and $20 \mathrm{~mL} \mathrm{HF}$ for $3.5 \mathrm{hr}$. After etching, the material was precipitated by centrifugation for $5 \mathrm{~min}$ at $8000 \mathrm{rpm}$. The supernatant containing HF was removed with a pipette, and the precipitate was redispersed in 20 $\mathrm{ml}$ of ethanol and then centrifuged again. This washing process was repeated again with $20 \mathrm{ml}$ of ethanol and once more with $20 \mathrm{ml}$ of chloroform. The resulting H-terminated nanocrystals were redispersed in $20 \mathrm{~mL}$ of 1-octene and then injected with a syringe into a 3-neck flask connected to a Schlenk line under vacuum. Liquid nitrogen was used to immediately cool the flask contents under vacuum, and then the dispersion was thawed to room temperature under nitrogen flow. The freeze-thaw process was repeated three more times. The dispersion was stirred under nitrogen flow at $120^{\circ} \mathrm{C}$ for $12 \mathrm{hr}$. The unpassivated nanocrystals were precipitated by 
centrifugation at $8000 \mathrm{rpm}$ for $5 \mathrm{~min}$. The passivated nanocrystals in octene were placed on a rotary evaporator under vacuum at $60^{\circ} \mathrm{C}$ to evaporate the octene. The nanocrystals were then redispersed in $3 \mathrm{ml}$ of hexanes. The Si nanocrystals were then purified by four washing steps using hexanes/ethanol as the solvent/antisolvent pair. The nanocrystals were redispersed in chloroform at a concentration of $6.75 \mathrm{mg} / \mathrm{ml}$ for use. The nanocrystals had a diameter measured by TEM of approximately $2.8 \mathrm{~nm}$ (See Supporting information Figure S1). By TGA, the prepared nanocrystals had a mass ratio of $37 \%$ silicon core to $63 \%$ capping ligand (See Supporting Information Figure S2).

Gold Nanocrystal Synthesis. Gold nanocrystals were synthesized using the procedures described in Rasch, et al. ${ }^{14} 33 \mathrm{mg}$ of gold (III) chloride trihydrate was dissolved in $20 \mathrm{~mL}$ of deionized water. A separate solution of $6 \mathrm{~g}$ of TOAB dissolved in $80 \mathrm{~mL}$ toluene was prepared. The aqueous and toluene solutions were mixed and stirred for $1 \mathrm{hr}$. The aqueous phase was discarded. 0.6 $\mathrm{mL}$ of 1-dodecanethiol was added to the toluene solution and stirred for $15 \mathrm{~min}$. An aqueous solution of $378 \mathrm{mg}$ of sodium borohydride in $20 \mathrm{ml}$ of water at $0{ }^{\circ} \mathrm{C}$ was prepared and added to the toluene solution. After stirring overnight, the aqueous phase was discarded. The nanocrystals were precipitated by adding $5 \mathrm{~mL}$ of the gold nanocrystal dispersion to a glass centrifuge tube and $20 \mathrm{~mL}$ of ethanol and centrifuging for $5 \mathrm{~min}$ at $8000 \mathrm{rpm}$. The supernatant was discarded and the precipitate was redispersed in toluene. This washing procedure was repeated until the entire gold solution had been washed with ethanol. Finally, the nanocrystals were redispersed and stored in toluene. Prior to use, the nanocrystals were dried and redispersed in chloroform at a concentration of $3 \mathrm{mg} / \mathrm{mL}$. The average diameter of the Au nanocrystals determined by TEM was $1.8 \mathrm{~nm}$ (Supporting Information, Figure S10a). The mass fraction of ligand in the sample was determined by TGA to be 28.5\% (Supporting Information, Figure S11). 
Cholesterol-CTAB Quatsomes Synthesis. Quatsomes were made by DELOS-SUSP (depressurization of an expanded liquid organic solution-suspension) as described in Ref. 24 and Ref. 25. $\mathrm{CO}_{2}$-expanded ethanol was used to dissolve cholesterol (chol). A $7.5 \mathrm{~mL}$ high-pressure vessel was loaded with a solution of $76 \mathrm{mg}$ of chol in $2.88 \mathrm{~mL}$ of ethanol at atmospheric pressure and $35^{\circ} \mathrm{C} . \mathrm{CO}_{2}$ was then added by syringe pump until reaching a working pressure of $10 \mathrm{MPa}$ and a $\mathrm{CO}_{2}$ molar fraction of $\mathrm{X}_{\mathrm{CO} 2}=0.62$. The system was kept at $35^{\circ} \mathrm{C}$ and $10 \mathrm{MPa}$ for approximately $1 \mathrm{hr}$. Finally, the $\mathrm{CO}_{2}$-expanded chol solution was removed from the reactor through a depressurization valve and collected in $24 \mathrm{~mL}$ of water with $72 \mathrm{mg}$ of dissolved CTAB to produce the chol-CTAB quatsomes. In this final step, a flow of $\mathrm{N}_{2}$ is used as a plunger to push down the $\mathrm{CO}_{2}$-expanded solution from the vessel and to maintain a constant pressure inside the vessel during depressurization. The molar ratio between the CTAB and the chol in the final formulation was 1 to 1 , which has been shown to be the correct proportion in order to have a pure vesicular phase. ${ }^{22}$ The final quatsome dispersions were composed of $7 \mathrm{mM}$ each of chol and CTAB in water containing $10 \%$ ethanol. This quatsome formulation is referred to throughout as “7 mM quatsomes.” A more diluted quatsome formulation composed of $0.7 \mathrm{mM}$ chol and CTAB was prepared by dilution of $7 \mathrm{mM}$ quatsomes with water with $10 \%$ ethanol. These are referred to as "0.7 mM quatsomes."

Preparation of Si nanocrystal/quatsome assemblies. In a typical procedure, $750 \mu \mathrm{L}$ of $7 \mathrm{mM}$ or $0.7 \mathrm{mM}$ quatsomes were added to a $2 \mathrm{~mL}$ glass vial. $20 \mu \mathrm{L}$ of a dispersion of $\mathrm{Si}$ nanocrystals in chloroform with a concentration of $6.75 \mathrm{mg} / \mathrm{mL}$ were added (corresponding to $0.135 \mathrm{mg}$ Si nanocrystals). The vial was bath-sonicated for $5 \mathrm{~min}$ at room temperature (Misonix 1510R-MT or Bransonic M1800 bath sonicator, 40kHz, 1/2 gallon tank). In experiments 
performed with only CTAB in place of the quatsomes, $7 \mathrm{mM}$ CTAB in water was bath sonicated with the same quantity of Si nanocrystals as above.

Liposome Preparation. DOPC (neutral) liposomes were prepared using the DELOSsusp process. ${ }^{23-25} 35 \mathrm{mg}$ of DOPC were dissolved in $1.9 \mathrm{~mL}$ of ethanol and loaded into a $7.5 \mathrm{~mL}$ high-pressure vessel at atmospheric pressure and $35^{\circ} \mathrm{C}$. The solution was then expanded with compressed $\mathrm{CO}_{2}$ until reaching a molar fraction of $\mathrm{CO}_{2}$ of $\mathrm{X}_{\mathrm{CO} 2}=0.76$ at a working pressure of $10 \mathrm{MPa}$. The system was kept at $35^{\circ} \mathrm{C}$ and $10 \mathrm{MPa}$ for $1 \mathrm{hr}$. Finally, the $\mathrm{CO}_{2}$-expanded DOPC solution was removed from the reactor through a depressurization valve and collected in $9 \mathrm{~mL}$ of an aqueous solution to create the liposomes. The final concentration of DOPC in the liposomal system was $3.6 \mathrm{mM}$ in high purity water containing $17 \%$ ethanol.

DOPG (anionic) liposomes were prepared by thin film hydration and extrusion. $7 \mu \mathrm{mol}$ DOPG in chloroform were dried into a film on a rotary evaporator for $15 \mathrm{~min}$. Residual solvent was removed by placing the film in a vacuum oven for $2 \mathrm{hr}$. The lipid film was then hydrated with $1 \mathrm{~mL}$ of $10 \mathrm{mM}$ HEPES, $10 \mathrm{mM} \mathrm{NaCl}$ and bath sonicated for $30 \mathrm{~min}$. Finally, the suspension was extruded 21 times through a polycarbonate filter (100 nm pore size) on an Avanti MiniExtruder (Avanti Polar Lipids).

Dialysis. Dialysis was performed using SnakeSkin Dialysis Tubing with $10 \mathrm{kDa}$ molecular weight cutoff (Life Technologies). Six rounds of dialysis into water, each lasting 24 hours, were performed.

Material Characterization. Transmission electron microscopy (TEM) and cryogenic transmission electron microscopy (cryo-TEM) images were acquired digitally using an FEI Tecnai Biotwin TEM operated at $80 \mathrm{kV}$ accelerating voltage. For imaging, Si nanocrystals were drop cast from chloroform onto 200 mesh carbon-coated copper grids (Electron Microscopy 
Science). Cryo-TEM samples were prepared using a Leica EM GP by dropping $3 \mu \mathrm{L}$ of sample onto Quantifoil R1.2/1.3 holey carbon on 300 mesh copper grids (Electron Microscopy Science) inside the environmental control chamber set to $25^{\circ} \mathrm{C}$ and $90 \%$ humidity. After a blotting time of $3.5 \mathrm{sec}$, the grid was plunged into liquid ethane to vitrify the sample. The grid was transferred to a Gatan 626 Cryo-Transfer Holder under liquid nitrogen. FEI low dose software was used to obtain images of cryo-TEM samples using a reduced electron beam dose.

Optical absorbance spectra were acquired on a Varian Cary 50 Bio UV-vis spectrophotometer or a Varian Cary 500 ultraviolet-visible-near infrared spectrophotometer. Photoluminescence (PL) emission and excitation (PLE) spectra were acquired on a Varian Cary Eclipse fluorescence spectrophotometer. Samples were diluted 1/20 prior to measurement. PL quantum yields were determined using Rhodamine B as a PL dye standard (see Supporting Information).

Dynamic light scattering (DLS) data were acquired on a Zetasizer Nano ZS (Malvern Instruments). Samples were measured in $40 \mu \mathrm{L}$ disposable cuvettes at $173^{\circ}$, with temperature set to $25^{\circ} \mathrm{C}$. All measurements were conducted in triplicate. Zetasizer software (Malvern Instruments) was used to analyze data and determine the average size (z-ave) and polydispersity index (PDI), with the dispersant set depending on the medium, as follows: $10 \%$ ethanol in water by volume had $8.06 \%$ by weight ethanol in water with viscosity of $1.28 \mathrm{cP}$ and refractive index of 1.34 , while $17 \%$ ethanol in water by volume had $13.9 \%$ by weight ethanol in water with a viscosity of $1.66 \mathrm{cP}$ and refractive index of 1.34 .

Thermal gravimetric analysis (TGA) was performed using a Mettler Toledo TGA/DSC 1. Nanocrystals dispersed in chloroform were dried in a $70 \mu \mathrm{L}$ alumina crucible (Mettler Toledo). 
Sample were heated under $50 \mathrm{~mL} \min ^{-1}$ of air flow at a rate of $10^{\circ} \mathrm{C} \min ^{-1}$ from $25^{\circ} \mathrm{C}$ to $800^{\circ} \mathrm{C}$ and then held at $800^{\circ} \mathrm{C}$ for $30 \mathrm{~min}$.

Molecular Dynamics Simulations. Molecular Dynamics (MD) simulations were performed using the NAMD 2.9 (CUDA version) software. ${ }^{30}$ Simulations were performed for Si and Au nanocrystals with their respective capping molecules in two different solvents (water and chloroform) at a temperature of $25^{\circ} \mathrm{C}$ and pressure of $1 \mathrm{~atm}$. The Si core contained $342 \mathrm{Si}$ atoms (to match the experimentally determined average diameter of $2.8 \mathrm{~nm}$ ) and 242 capping molecules (1-octene). The Au core had $281 \mathrm{Au}$ atoms (to match the experimentally determined average diameter of $1.8 \mathrm{~nm}$ ) and 65 capping molecules (1-dodecanethiol). This corresponds to a surface coverage of 9.8 molecules $/ \mathrm{nm}^{2}$ for the Si particle and 6.4 molecules $/ \mathrm{nm}^{2}$ for the Au particle. The interactions between atoms were modeled using the standard CHARMM force field for Si, Au and water and the CGenFF version of the CHARMM force field ${ }^{31}$ for chloroform, 1-octene and 1-dodecanethiol. More details are provided as Supporting Information.

\section{RESULTS AND DISCUSSION}

Incorporation of Silicon Nanocrystals into Cholesterol-CTAB Quatsomes. Silicon nanocrystals with an average core diameter of $2.8 \pm 0.6 \mathrm{~nm}$ with octene capping ligands dispersed in chloroform were incorporated into pre-formed aqueous $7 \mathrm{mM}$ quatsomes using a 5 minute bath sonication procedure, as outlined in Figure 1a. The quatsome dispersions initially have a cloudy blue/grey appearance and then become light yellow following the addition of the Si nanocrystals and bath sonication. There is no visible precipitation or phase separation after addition of the nanocrystals to the quatsomes. Figures $1 \mathrm{~b}-1 \mathrm{~d}$ show photographs of the quatsome and nanocrystal dispersions before and after they are combined, along with TEM images. The 
nanocrystal quatsome assemblies dispersed in water exhibit orange fluorescence characteristic of Si nanocrystals dispersed in chloroform, as shown in Figures 1c and 1d. There was no observable fluorescence when Si nanocrystals were added to water without the presence of quatsomes, as the nanocrystals have a hydrophobic ligand shell and do not disperse in water.
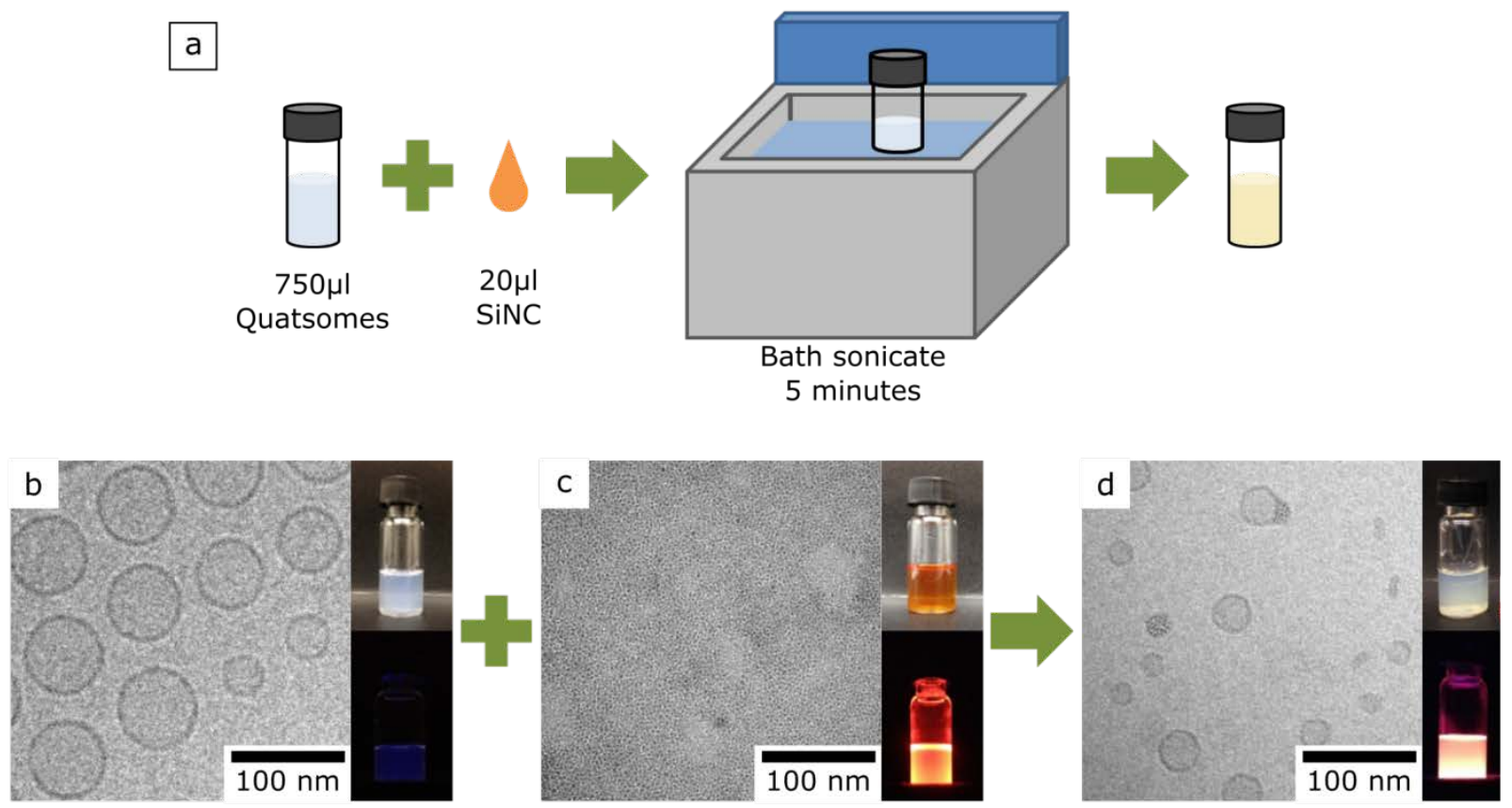

Figure 1. (a) Schematic representation of the process for incorporating Si nanocrystals into quatsomes through bath sonication. CryoTEM (b and d) and TEM (c) images of $7 \mathrm{mM}$ quatsomes, Si nanocrystals, and their assemblies along with photographs of the dispersions under ambient light (on the top) and a $365 \mathrm{~nm}$ UV lamp (on the bottom). Diagrams of the three structures are also shown in the bottom right.

Figure 2 shows additional cryoTEM images of the fluorescent Si nanocrystal-quatsome assemblies. The Si nanocrystals appear as clusters of similar size as the quatsomes. The nanocrystals do not distribute in the surfactant bilayers of the quatsomes as dodecanethiol- 
capped gold nanocrystals have been observed in other liposomal vesicles. ${ }^{14,18}$ The Si nanocrystal clusters are attached to one (green arrow) or more quatsomes (blue arrow), or in some cases not attached to any quatsomes (red arrow).
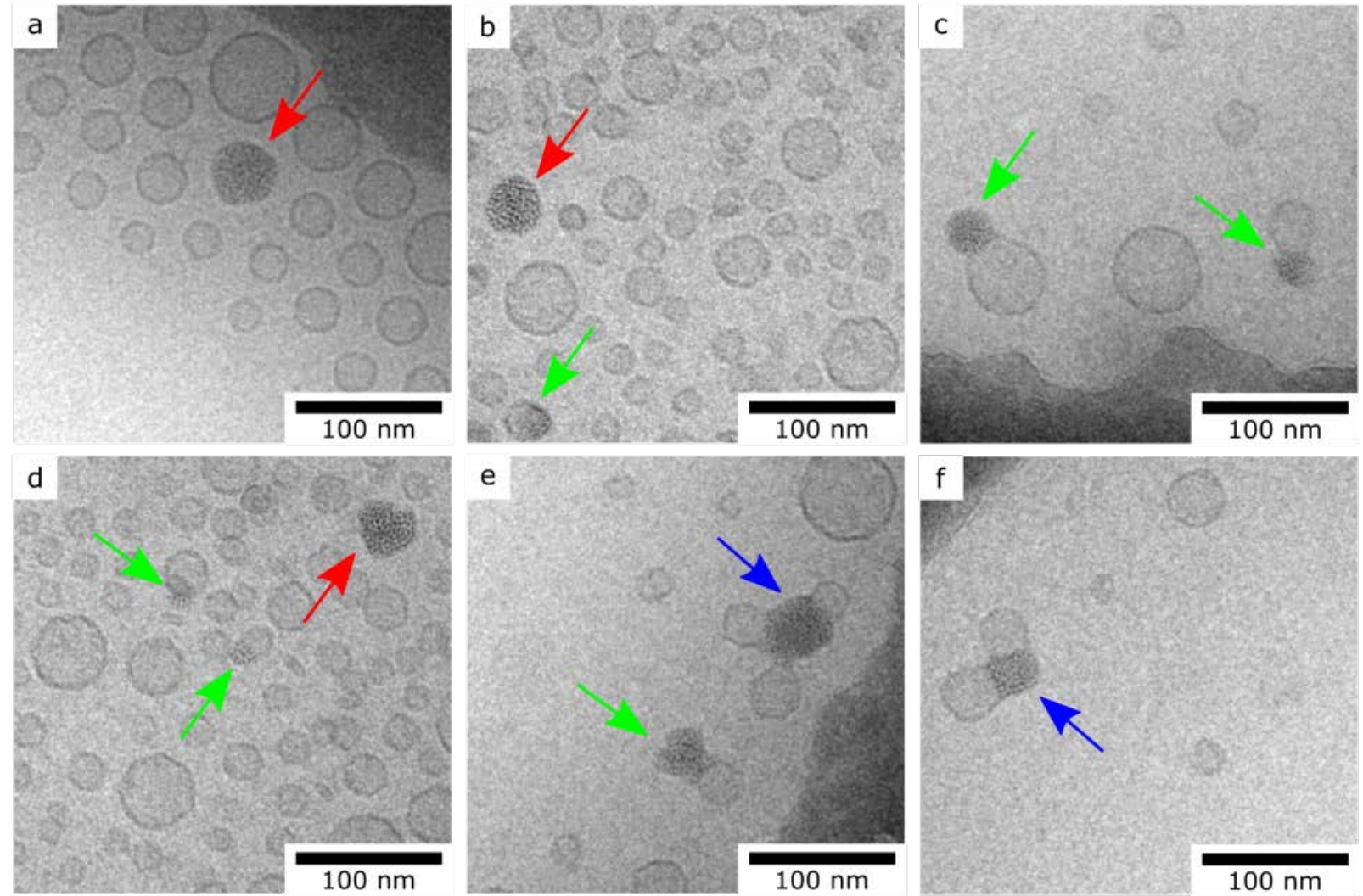

Figure 2. CryoTEM images of fluorescent dispersions of Si nanocrystal-7mM quatsome assemblies. The red arrows indicate $\mathrm{Si}$ nanocrystal clusters that are not associated with quatsomes; whereas, the green and blue arrows show clusters associated with one or more quatsomes, respectively.

Figure 3 compares the optical absorbance, PLE and PL spectra collected from Si nanocrystals dispersed in chloroform and associated with quatsomes in aqueous media prepared with the same nanocrystal concentration. The spectra are nearly identical, indicating that the 
optical properties of the nanocrystals are successfully transferred to aqueous media using quatsomes as a dispersing vehicle. The quatsome-Si nanocrystal preparations have slightly higher absorbance, which results from additional light scattering of the quatsomes (see, for example, the turbidity of the quatsome dispersions in Figure 3). The PL intensity of the quatsome-Si nanocrystal assemblies was lower than the Si nanocrystals dispersed in chloroform, although the samples still exhibit relatively bright fluorescence when placed over a UV lamp. The decreased PL is partly due to the light scattering of the quatsomes. The PL peak positions of both samples were also nearly identical. The PL quantum yields of the Si nanocrystals in chloroform and in the quatsome assemblies in water were $16.1 \%$ and $6.6 \%$, respectively (see Supporting Information for quantum yield calculation).

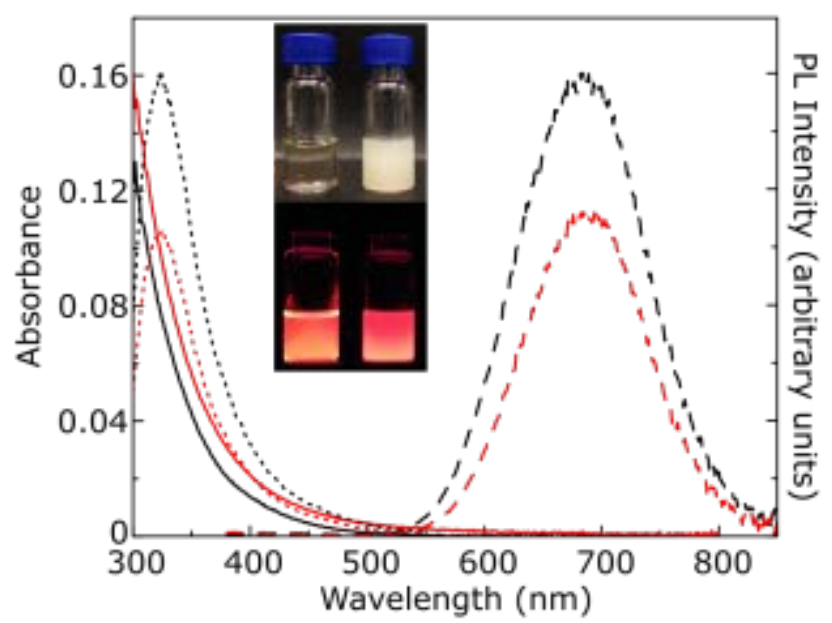

Figure 3. Absorbance (solid lines), PL $\left(\lambda_{\mathrm{exc}}=320 \mathrm{~nm}\right)$ (dashed lines), and PLE $\left(\lambda_{\mathrm{em}}=660\right)$ (dotted lines) spectra for octane-passivated $\mathrm{Si}$ nanocrystals dispersed in chloroform (black) and incorporated into quatsomes in aqueous media (red). Each sample contained $0.135 \mathrm{mg}$ of $\mathrm{Si}$ nanocrystals in a total volume of $0.77 \mathrm{~mL}$ of chloroform or quatsomes, and was diluted $1 / 20$ for the measurements. Inset: photographs of vials of (left) Si nanocrystals in chloroform and (right) 
quatsome-Si nanocrystal assemblies, each containing the same concentration of Si nanocrystals, under ambient light (top) or illuminated with a $365 \mathrm{~nm}$ ultraviolet lamp (bottom).

Colloidal and Fluorescence Stability of Si Nanocrystal-Quatsome Assemblies. The colloidal and fluorescence stability of the quatsome-Si nanocrystal assemblies was tested. Figure 4 shows photographs of $7 \mathrm{mM}$ quatsome-Si nanocrystal dispersions over the course of 12 weeks. The dispersions were visibly stable during this time period and retained their fluorescence. The optical absorbance did decrease during the first two weeks, but then appeared to stabilize. Perhaps the system is not completely at equilibrium initially, or perhaps there is some oxidation of poorly capped nanocrystals during this time period. Surface oxidation as a result of the aqueous environment may also contribute to the decrease in the average PL wavelength as the nanocrystal core size shrinks. For comparison, over 8 weeks the average PL wavelength blue shifted by $40 \mathrm{~nm}$ for the quatsome-Si nanocrystal assemblies but only by $3 \mathrm{~nm}$ for SiNCs in chloroform, suggesting that the aqueous environment and/or CTAB and cholesterol molecules contribute to the observed blue shift. There is no observable change in the morphology of the assemblies imaged by CryoTEM (Figure 5). 

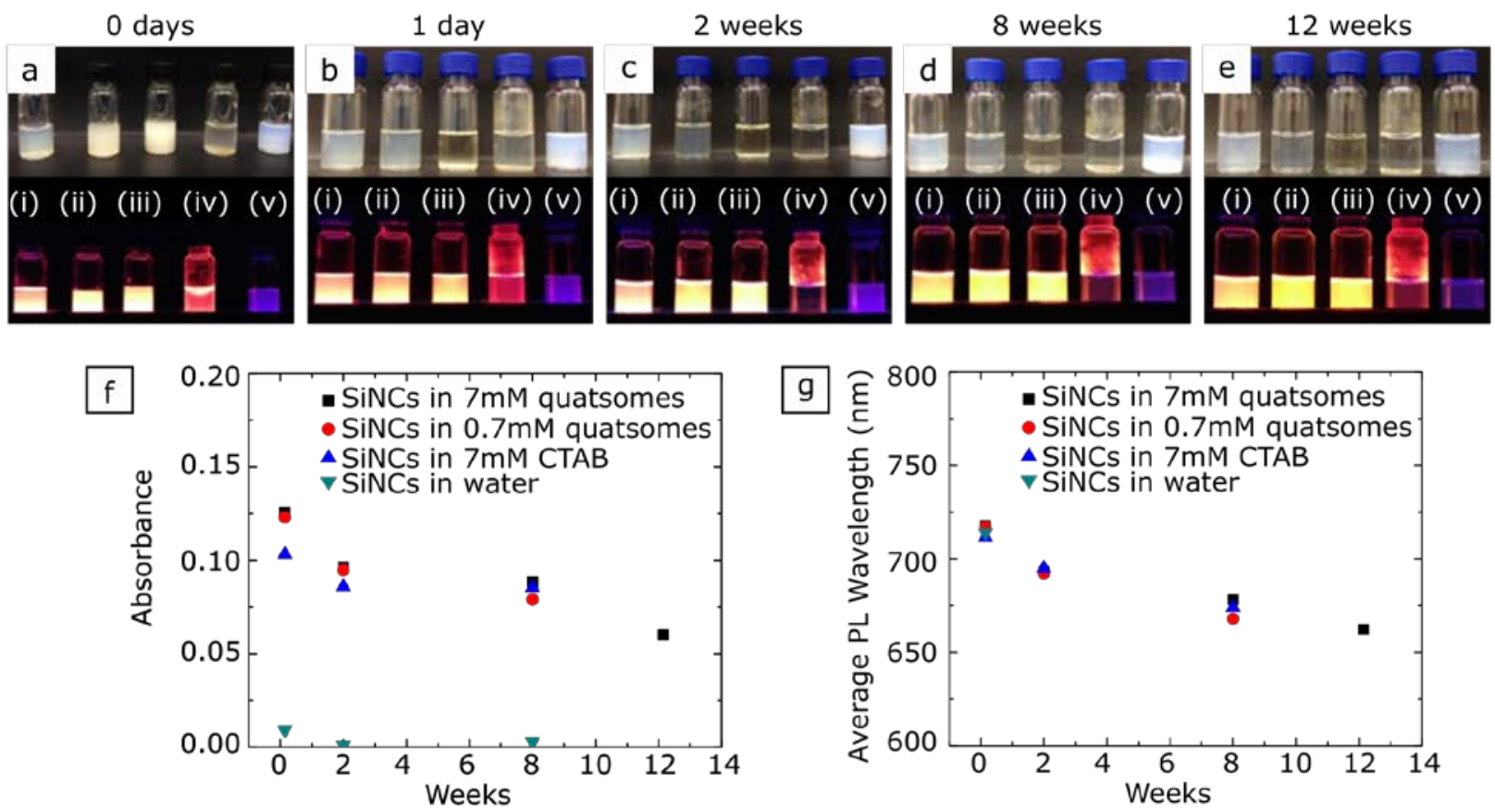

Figure 4. Fluorescence and colloidal stability of Si nanocrystals dispersed in aqueous solution in using quatsomes or CTAB. The five samples shown in (a-e) are (i) - $7 \mathrm{mM}$ quatsome-Si nanocrystal assemblies (with CTAB concentration above cmc), (ii) $\bullet 0.7 \mathrm{mM}$ quatsome-Si nanocrystals assemblies (with CTAB concentration bellow $\mathrm{cmc}$ ), (iii) $\Delta$ SiNCs in $7 \mathrm{mM} \mathrm{CTAB}$ micelles, (iv) $\nabla$ SiNCs in $10 \% \mathrm{EtOH}$, and (v) chloroform in $7 \mathrm{mM}$ CTAB quatsomes. Vials were prepared by adding $20 \mu \mathrm{l}$ SiNC (in chloroform) to $0.75 \mathrm{ml}$ of solution, and then bath sonicated for 5 minutes. Photographs of vials under ambient light (top) and on a $365 \mathrm{~nm} \mathrm{UV}$ lamp (bottom) taken (a) immediately, (b) one day, (c) 2 weeks, (d) 8 weeks, and (e) 12 weeks after preparation. Absorbance (f) measured at $320 \mathrm{~nm}$ for samples with SiNCs and average PL wavelength (g) for samples with SiNCs (excited at $320 \mathrm{~nm}$ ) demonstrate the stability of the nanoparticle fluorescence in quatsomes. 

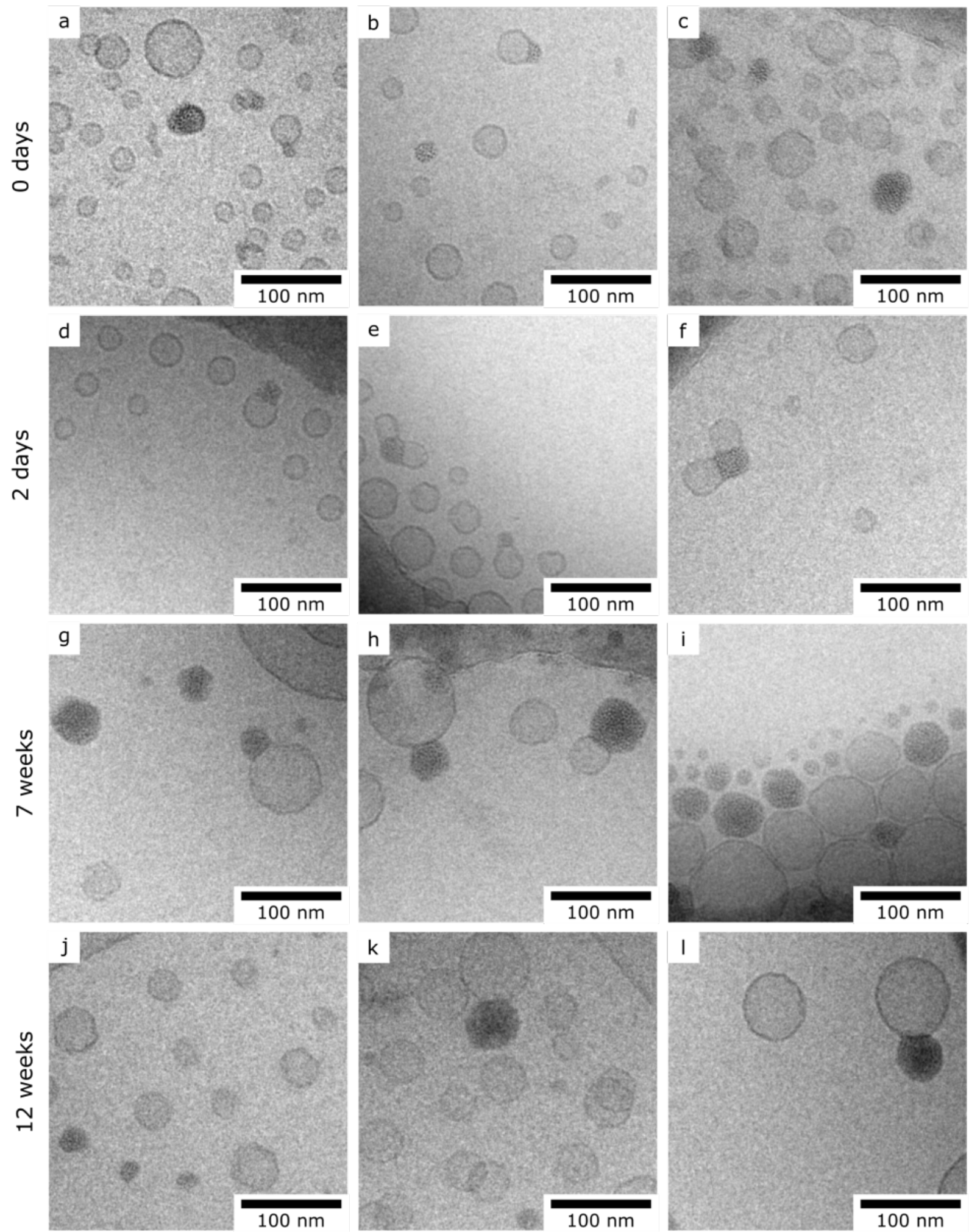
Figure 5. CryoTEM images of $7 \mathrm{mM}$ quatsome-Si nanocrystal assemblies taken (a)-(c) on the day the sample was prepared and (d)-(f) 2 days, (g)-(i) 7 weeks, and (j)-(l) 12 weeks after the sample was prepared.

The $7 \mathrm{mM}$ quatsome-Si nanocrystal assemblies were also monitored by DLS (Figure 6). There was no observable change in the mean size of the assemblies, but the size distribution became narrower, with the polydispersity index (PDI) decreasing from $0.262 \pm 0.006$ to $0.157 \pm$ 0.008 over 8 weeks. A similar narrowing of the size distribution was also observed for quatsomes that were bath-sonicated without any nanocrystals (sample v), indicating that this narrowing of the size distribution is inherent to the quatsomes and not related to the presence of the nanocrystals. The sonication parameters were also found to influence the Si nanocrystalquatsome assembly size and polydispersity (See Supporting Information). For consistency, five minutes of sonication time was used throughout. A similar peak narrowing phenomena was observed in vesicle assemblies formed by cholesterol and zwitterionic surfactants. ${ }^{32,33}$ Over time the distribution narrows as the quatsomes return to a thermodynamically stable size range, as expected for stable vesicular assemblies. ${ }^{34}$ 

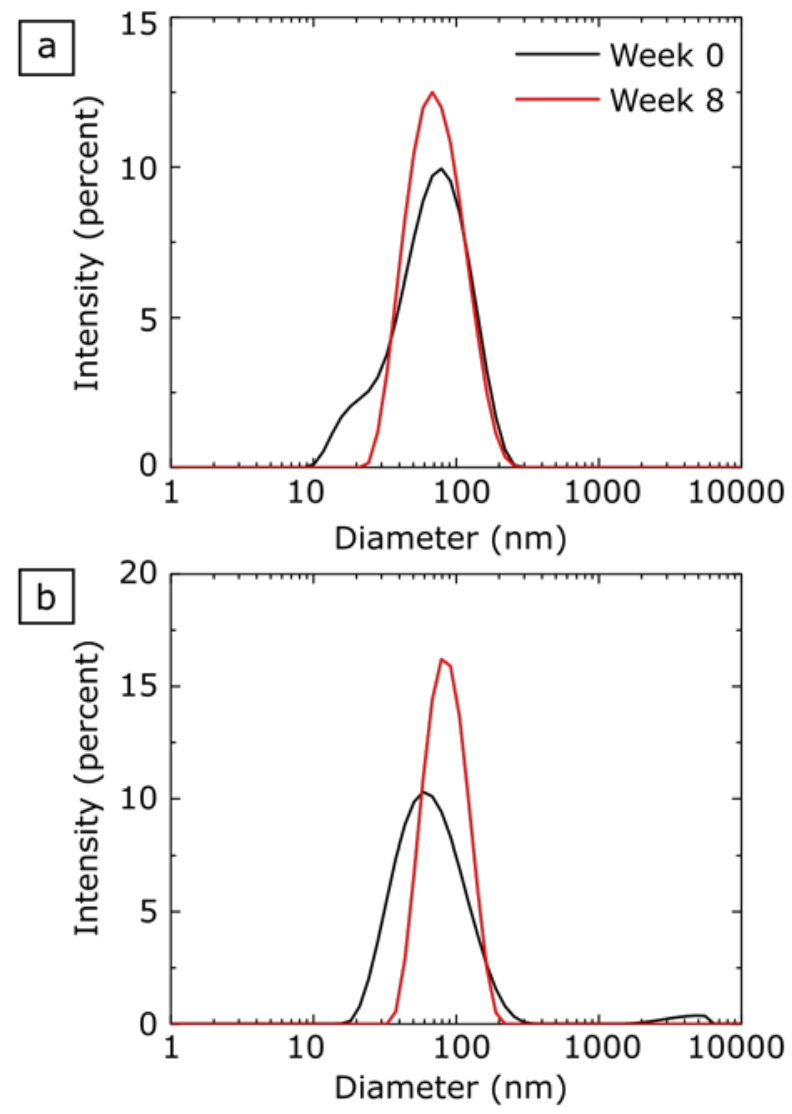

Figure 6. Monitoring of the change in size distribution by DLS of (a) 7mM quatsome-Si nanocrystal assemblies and (b) nanocrystal-free quatsomes. Black curves correspond to measurements done in week 0 , red curves correspond to measurements done in week 8 . After 8 weeks of annealing, the quatsome size distributions became narrower around a preferred size. (a) $20 \mu \mathrm{L}$ of SiNCs in chloroform incorporated into $750 \mu \mathrm{L}$ quatsomes (sample (i) from stability trials). Sizes measured by DLS (each measured in triplicate) were: week 0 z-ave $54.3 \pm 0.4 \mathrm{~nm}$, PDI $0.262 \pm 0.006$; week 8 z-ave $65.2 \pm 0.2 \mathrm{~nm}$, PDI $0.157 \pm 0.008$. (b) $20 \mu \mathrm{l}$ of chloroform with $750 \mu \mathrm{l}$ quatsomes (sample (v) from stability trials). Sizes measured by DLS (each measured in triplicate) were: week 0 z-ave $58.4 \pm 0.7 \mathrm{~nm}$, PDI $0.235 \pm 0.008$; week 8 z-ave $79.3 \pm 0.3 \mathrm{~nm}$, PDI $0.10 \pm 0.02$ 
The stability of the quatsome-Si nanocrystal assemblies was also tested after dilution. As shown in Figure 4, the assemblies were still stable after a 10X dilution, i.e., $0.7 \mathrm{mM}$ quatsome-Si nanocrystals. This is an important result, as it indicates that there is no loss of integrity of the assemblies even when the CTAB concentration is below the CMC of free CTAB. Figure 7 shows cryoTEM images of the $0.7 \mathrm{mM}$ quatsome-Si nanocrystal assemblies, which exhibit similar morphology as the $7 \mathrm{mM}$ quatsome-nanocrystal assemblies. Indeed in many medical applications, dilution is required and it is important that the quatsome-nanocrystal assemblies retain their colloidal stability under these conditions. This is not the case when Si nanocrystals are dispersed only with CTAB. As shown in Figure 4, CTAB could also be used to form stable dispersions of Si nanocrystals in aqueous media; however, with pure CTAB it is not possible to stabilize Si nanocrystals below the CMC. The CTAB-dispersed Si nanocrystals also tended to produce very polydisperse aggregates of nanocrystals, as observed by CryoTEM (Figure 8). Again, for medical applications, consistent size and concentration of load delivery is important, and thus the wide size distribution observed with CTAB micelles would not be ideal for those applications. This is discussed in more detail below. 

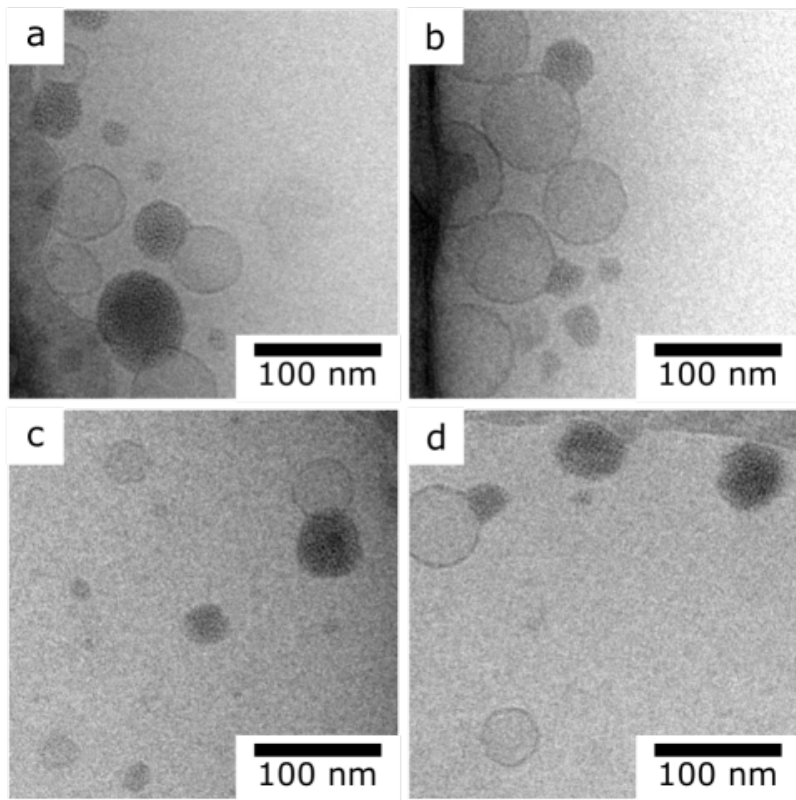

Figure 7. CryoTEM of $0.7 \mathrm{mM}$ quatsome-Si nanocrystal assemblies. The CTAB concentration in these assemblies is below the CMC of free CTAB.
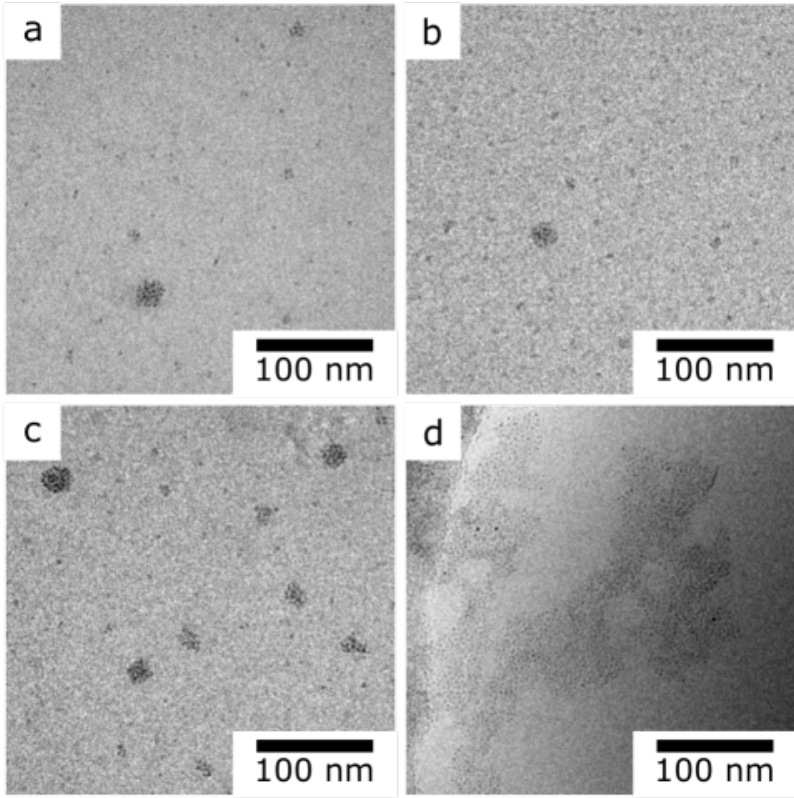

Figure 8. CryoTEM images of Si nanocrystals dispersed with $7 \mathrm{mM} \mathrm{CTAB}$ (above CMC of free CTAB). 
Comparison to Liposomal Structures. For comparison to liposomal vesicles, Si nanocrystals were combined with charge-neutral DOPC and anionic DOPG lipids—systems that have been used in the past to disperse dodecanethiol-capped gold nanocrystals. ${ }^{14,18}$ It should be pointed out, that whereas quatsomes are extremely thermodynamically stable vesicular structures, the stability of liposomes is kinetically limited and the collapsed planar lamellar is the equilibrium state of aggregation. ${ }^{22}$ The same bath sonication used to make the nanocrystalquatsome assemblies was applied to Si nanocrystal incorporation into the DOPC and DOPG liposomes. Figure 9 shows cryoTEM images of these nanocrystal-liposome assemblies. The charge-neutral DOPC liposomes become multilamellar, mixed with large aggregates of $\mathrm{Si}$ nanocrystals (Figures 9a-9d). DLS of the DOPC liposomes showed that they had a z-average size of $58 \pm 2 \mathrm{~nm}$ (PDI $0.230 \pm 0.008$ ) before sonication and grew to a z-average of $464 \pm 28 \mathrm{~nm}$ (PDI $0.135 \pm 0.143$ ) after sonication in the presence of the Si nanocrystals. The vial containing the DOPC and Si nanocrystals also had a white precipitate of lipid at the bottom after sonication. The anionic DOPG liposomes retained their initial size, but no Si nanocrystals were observed to associate with the liposomes (Figures 9e-9h). The dispersion, however, was fluorescent and separate lipid-stabilized aggregates of Si nanocrystals were observed in the cryoTEM images. For both DOPC and DOPG liposomes, the dispersions remained fluorescent for at least 3 days. 

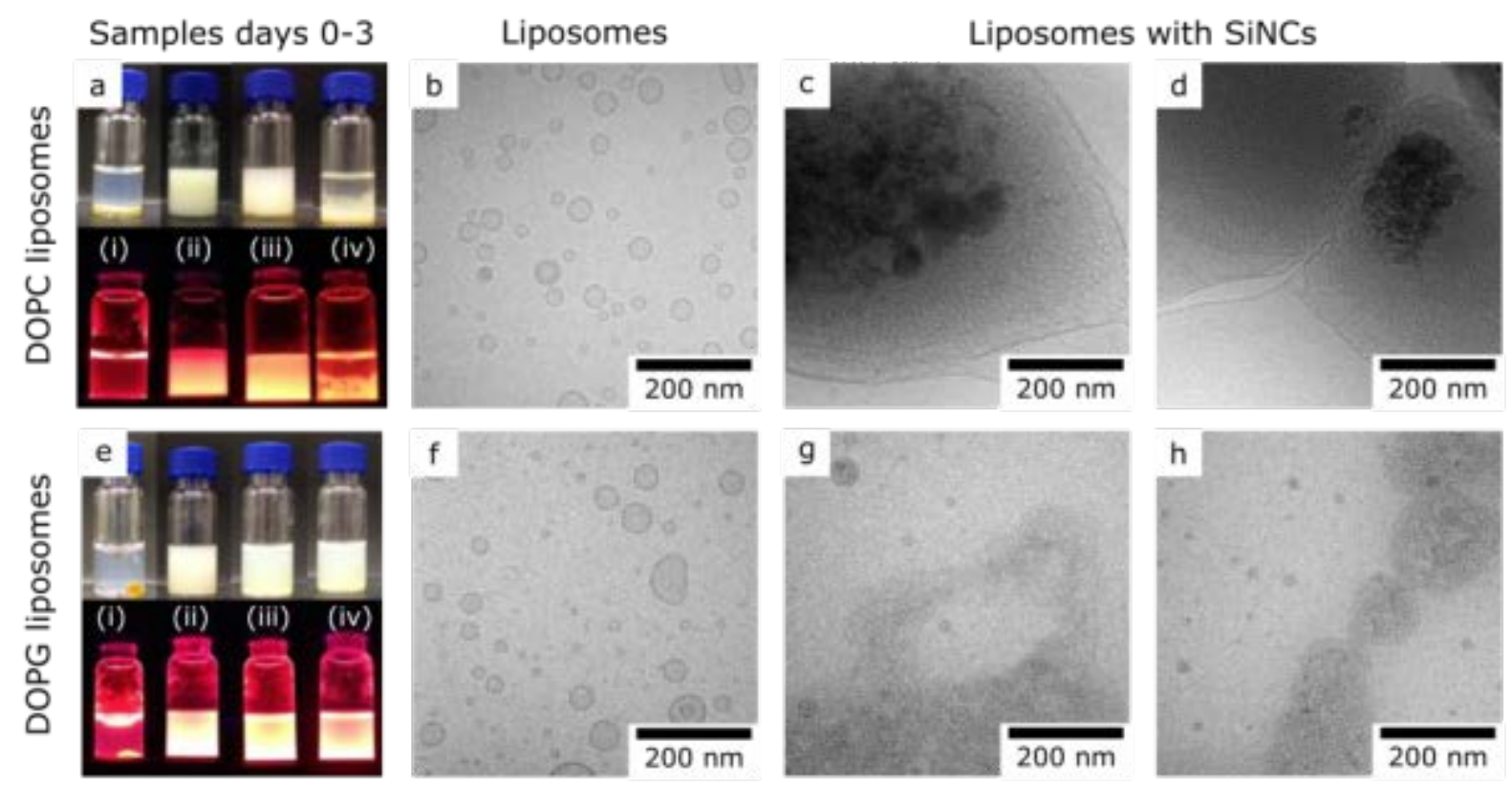

Figure 9. Results from preparing DOPC (neutral) and DOPG (anionic) liposomes separately and then adding $20 \mu \mathrm{l} \mathrm{SiNCs} \mathrm{in} \mathrm{chloroform} \mathrm{and} \mathrm{bath} \mathrm{sonicating} \mathrm{for} 5$ minutes. Photographs of vials of (a) DOPC and (e) DOPG were taken under ambient light and on a $365 \mathrm{~nm}$ UV lamp (i) before bath sonication, (ii) immediately after sonication, (iii) after 1 day, and (iv) after 3 days, showing that DOPC precipitated out of solution while the DOPG solution remained turbid. CryoTEM images of the liposomes before adding nanocrystals showed that the (b) DOPC and (f) DOPG liposomes formed small, predominantly unilamellar vesicles. CryoTEM images (c-d) of the DOPC liposomes after bath sonication showed that SiNCs incorporated with the lipid, however formed large complexes. CryoTEM images (g-h) of the DOPG liposomes after bath sonication showed that the SiNCs aggregated outside of empty liposomes, and no incorporation with the liposomes was observed.

Dispersion Stability of Diluted Assemblies of Si Nanocrystals and Quatsomes and CTAB Micelles. Medical diagnostic or theranostic applications that use fluorescent nanocrystals 
as contrast agents usually require dilution. Si nanocrystals were dispersed in CTAB micelles with $\mathrm{CTAB}$ at the same concentration as in the $7 \mathrm{mM}$ quatsome-Si nanocrystal dispersions. Both dispersions were then dialyzed to test their stability against dilution. Figure 10 compares the stability of the dispersions. Both dispersions are initially fluorescent, but only the quatsome dispersion remains fluorescent after dialysis. The CTAB surfactant is only weakly associated with the Si nanocrystals in the micelles, and repeated dilutions lead to loss of dispersibility of the nanocrystals. CryoTEM images (Figure 10f-10i) of the quatsome assemblies show that the dialysis has little effect on the structure of the assemblies. This shows the benefit of the quatsome assemblies compared to CTAB micellar dispersions of the nanocrystals: while both maintain fluorescence for 12 weeks (Figure 4), only the quatsome assemblies are stable under dilution.
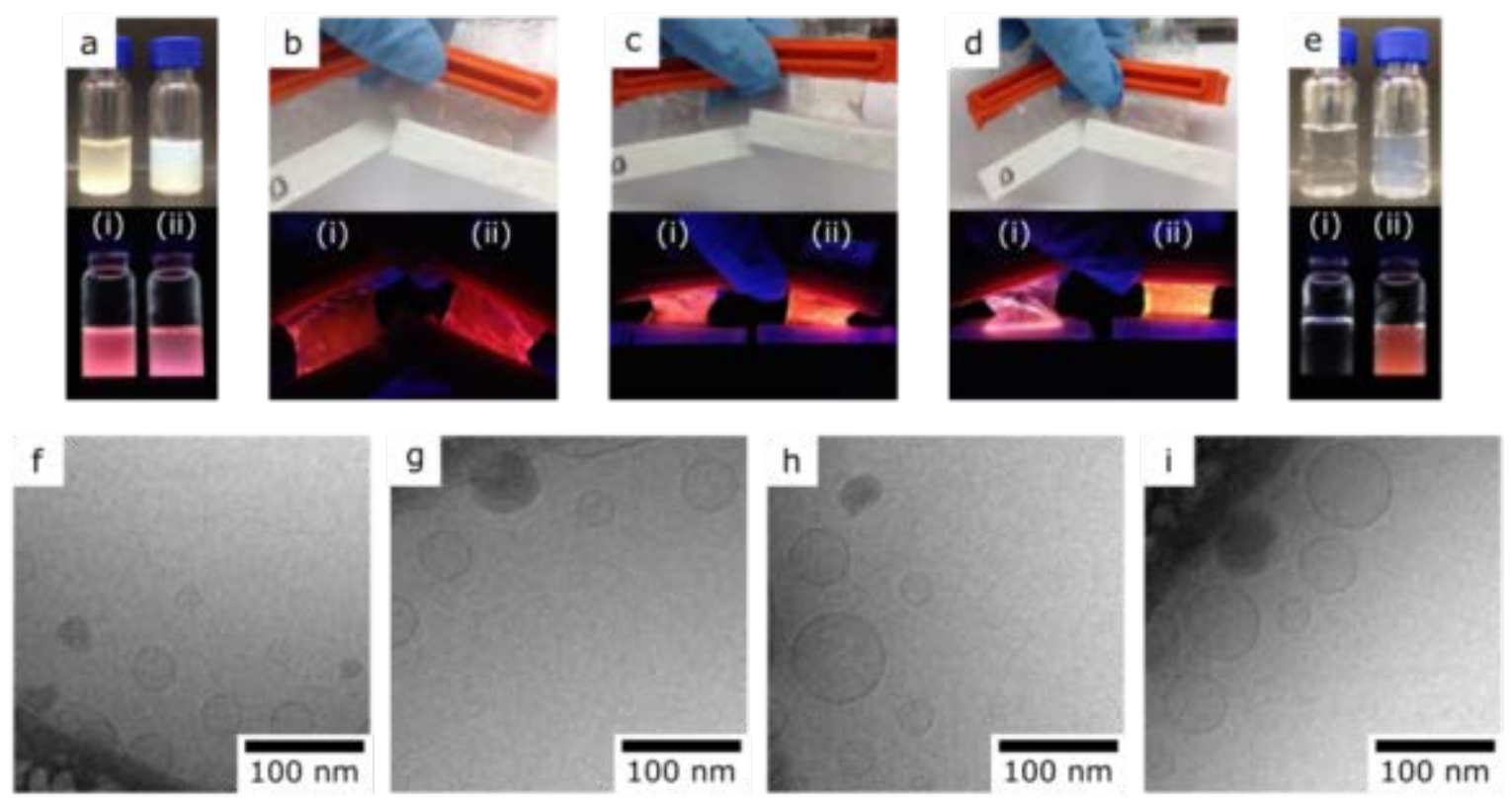

Figure 10. Dilution by dialysis of (i) Si nanocrystals dispersed with $7 \mathrm{mM}$ CTAB dispersed and (ii) $7 \mathrm{mM}$ quatsome-Si nanocrystal dispersions. (a) Photographs of vials containing (i) and (ii) one day after preparation under ambient light (top) and illumination with a $254 \mathrm{~nm}$ UV lamp 
(bottom). (b-d) Photographs of the nanocrystal dispersions in the dialysis after (b) 1, (c) 3, and (d) 6 rounds of dialysis, under ambient light (top) and illumination with a $254 \mathrm{~nm}$ UV lamp (bottom). (e) Photographs of dispersions removed from the dialysis tubing after 6 rounds of dialysis, under ambient light (top) and illumination with a $254 \mathrm{~nm}$ UV lamp (bottom). (f-i) CryoTEM images of the $7 \mathrm{mM}$ quatsome-Si nanocrystals after 6 rounds of dialysis.

\section{Role of chloroform in dispersing $\mathrm{Si}$ nanocrystals in quatsome assemblies.}

Fluorescent assemblies of quatsomes and $\mathrm{Si}$ nanocrystals were obtained when $750 \mu \mathrm{L}$ of quatsomes (at either $7 \mathrm{mM}$ or diluted to $0.7 \mathrm{mM}$ concentrations) were combined with $20 \mu \mathrm{L}$ of $6.75 \mathrm{mg} / \mathrm{mL}$ Si nanocrystals in chloroform (Figure 4). If a larger volume of chloroform was used with a lower concentration of Si nanocrystals, a white precipitate formed (Figure S7). The larger volume of chloroform destabilized the quatsomes. Conversely, if a smaller volume of chloroform was added with a higher concentration of Si nanocrystals, not all of the nanocrystals incorporated into the quatsomes and there was observably nanocrystal precipitation (Figure S7f). A small amount of chloroform enhances the uptake of dodecanethiol-capped Au nanocrystals into the lipid bilayer of DOPC liposomes. ${ }^{18}$ A similar role for chloroform might be occurring here in the Si nanocrystal-quatsome assemblies. The small $20 \mu \mathrm{L}$ volume of chloroform does not destabilize the quatsomes, as shown in Figure 5, sample v.

Other approaches to loading Si nanocrystals into the quatsomes were also explored, but bath sonication of a mixture of Si nanocrystals dispersed in chloroform added to a quatsome dispersion was found to be the only way to obtain fluorescent assemblies in the same size range as quatsomes. For example, direct addition of Si nanocrystals into the DELOS-susp quatsome formation process did not yield fluorescent dispersions. Probe sonication and vortexing of Si 
nanocrystals added to quatsome dispersions also did not yield fluorescent dispersions. Probe sonication led to aggregation, while the vortexed sample lost fluorescence (Supporting Information, Figure S6).

Interaction Between Dodecanethiol-capped Gold Nanocrystals and Quatsomes. We found that it was not possible to make stable assemblies of dodecanethiol-capped $\mathrm{Au}$ nanocrystals and quatsomes. Dispersions of $1.8 \mathrm{~nm}$ diameter dodecanethiol-capped gold nanocrystals in $20 \mu \mathrm{L}$ of chloroform ( $3 \mathrm{mg} / \mathrm{mL}$ Au nanocrystals) were combined with $750 \mu \mathrm{L}$ of $7 \mathrm{mM}$ quatsomes and bath sonicated for $5 \mathrm{~min}$. Immediately after sonication, the dispersions would appear cloudy with a grey hue, indicating at least partial dispersibility of the nanocrystals by the presence of quatsomes (Figures 11c-11d); however, the dispersions changed from grey to purple after only a few days and an plasmon peak in the optical absorbance spectra appeared at $\sim 520 \mathrm{~nm}$, indicating that the dispersion was unstable and that the nanocrystals had aggregated or coalesced. ${ }^{18}$ In comparison, Au nanocrystals readily incorporate into the lipid bilayer of phosphatidylcholine liposomes to form dispersions that are stable for weeks. ${ }^{14,18}$ CryoTEM of the purple quatsome-Au nanocrystal dispersions one week after sonication (Figures 11e-11f) showed that the nanocrystals were aggregated (Figures 11e and 11f) and apart from empty quatsomes. Additional CryoTEM (Figures 11g-11h) of the purple dispersion after several weeks showed that the nanocrystals had coalesced to form large particles approximately 5-10 nm in diameter that were not associated with quatsomes (the average size of these coalesced particles was found to be $6.7 \mathrm{~nm}$ with a standard deviation of $3.0 \mathrm{~nm}$, see Supporting Information). It is possible that—similar to Si nanocrystals dispersed with CTAB (Figure 8)—the coalesced gold nanoparticles remain dispersed due to an interaction with CTAB micelles. Further, CTAB is 
commonly used in the synthesis of gold nanocrystals in various sizes and shapes, ${ }^{35-37}$ and known to disperse and interact with Au colloids.
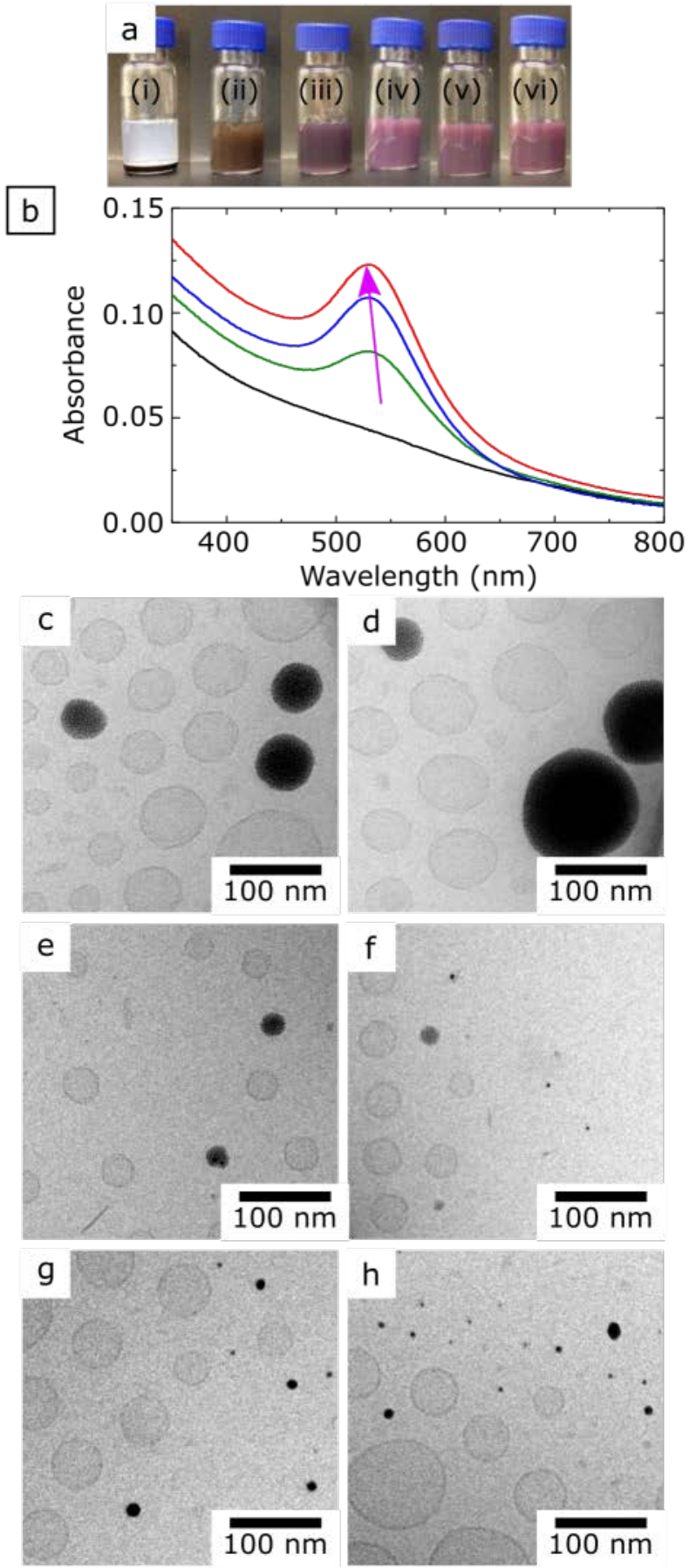

Figure 11. Results of incorporating $1.8 \mathrm{~nm}$ dodecanethiol capped AuNCs into quatsomes through 5 minutes of bath sonication $(0.75 \mathrm{ml}$ of $7 \mathrm{mM}$ quatsomes with $20 \mu \mathrm{l}$ of AuNC in 
chloroform). (a) Photographs of vial with AuNC and quatsomes over 8 weeks showed a gradual change in color from brown-grey to purple. From left to right: (i) before sonication, (ii) immediately after sonication, (iii) after 1 day, (iv) after 2 weeks, (v) after 4 weeks, and (vi) after 8 weeks. UV-Vis data for samples over time (b) showed increase in plasmon peak intensity (samples diluted 1/20 for measurements) (black line is 0 days, green line is 1 day, blue line is 4 weeks, and red line is 8 weeks). Cryo-TEM images of quatsome-AuNC dispersions at (c-d) 0 days, (e-f) 1 week, and (g-h) several weeks after preparation, showing the gradual decrease in aggregate size and formation of coalesced gold nanoparticles separate from empty quatsomes. Measurements of nanocrystal size indicated that while the gold nanocrystals in chloroform had an average size of $1.8 \mathrm{~nm}$ with a standard deviation of $0.4 \mathrm{~nm}$, several weeks after preparation the AuNC average size was $6.7 \mathrm{~nm}$ with a standard deviation of $3.0 \mathrm{~nm}$ (Supporting Information).

\section{Computer Simulations of Si and Au nanocrystals and proposed mechanism for} nanocrystal-quatsome interactions. All-atomic molecular dynamics simulations (MD) of the $\mathrm{Si}$ and Au nanocrystals were performed to help elucidate why $\mathrm{Au}$ and $\mathrm{Si}$ nanocrystals exhibit different association behavior with the quatsome. Figure 12 shows images of snapshots from the MD simulations of the Si and Au nanocrystals surrounded by water or chloroform. The structure of the ligand capping layers are noticeably different for the two types of nanocrystals. The ligand shell on the Si nanocrystals is a compact, solid-like layer of octane molecules, which form ordered patterns in both water and chloroform. In both cases, octane molecules covering SiNCs are found in an extended configuration at $94 \%$ of their maximum possible length, which corresponds to a fully extended chain. They are also oriented at a well-defined angle $\left(28^{\circ}\right)$ 
normal to the Si surface. In contrast, the 1-dodecanethiol ligands on the Au nanocrystals form a disordered, fluid-like, brush layer. The ligand layer is more disordered in chloroform than in water. The simulations indicated that 1-dodecanethiol molecules have an average length of $69 \%$ (in water) and 74\% (in chloroform) of their maximum length without any preferred orientation (in contrast with the case of 1-octene molecules capping SiNC).
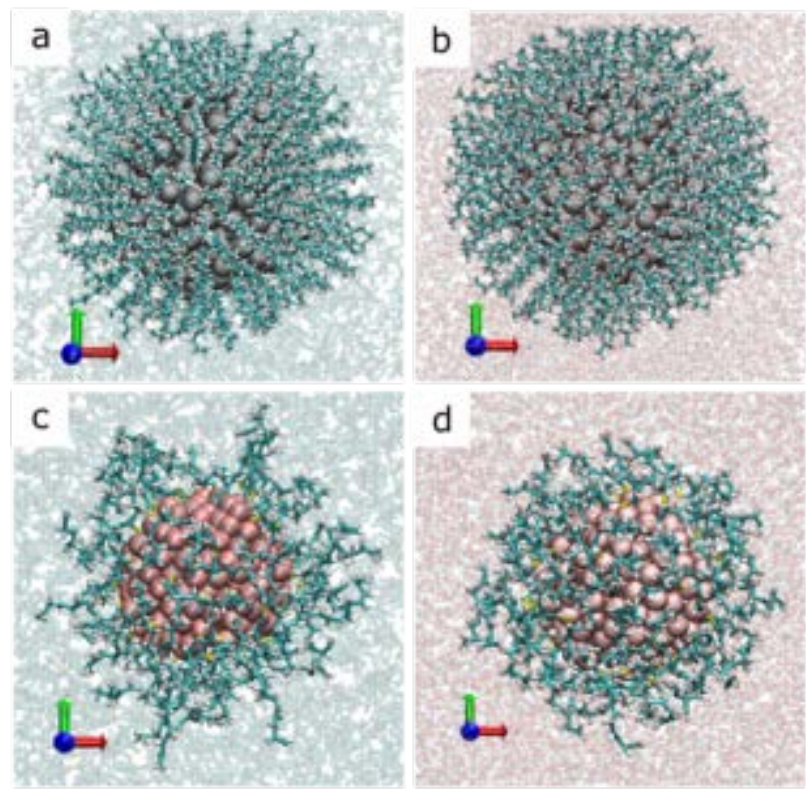

Figure 12. Snapshots from MD simulations using the Visual Molecular Dynamics (VMD) program. ${ }^{38}$ A Si nanoparticle capped with 1-octene molecules in (a) chloroform and (b) water. An Au nanoparticle capped with 1-dodecanethiol in (c) chloroform and (d) water. Si and Au atoms are shown with their Van der Waals radius. Capping molecules are shown in bond representation. Solvent molecules are drawn translucent. The results show that SiNCs have denser packed ligand layers than the AuNCs, and that in chloroform the ligands are more extended. 
The simulations also computed the thickness of the coating organic layer, which takes into account ligand lengths and their orientation (angle) with the surface (see Supporting Information for a complete description of the procedure). The thickness of the organic layer was $1.14 \mathrm{~nm}$ for Si nanocrystals in both water and chloroform, which results in a particle with a total diameter of $5.1 \mathrm{~nm}$. The thicknesses obtained for the layers of 1-dodecanethiol on $\mathrm{Au}$ nanocrystals were $0.89 \mathrm{~nm}$ and $1.14 \mathrm{~nm}$ in water and chloroform, respectively, which gives total particle diameters of $3.7 \mathrm{~nm}$ and $4.2 \mathrm{~nm}$, respectively. The simulations reveal that the $\mathrm{Si}$ and $\mathrm{Au}$ nanocrystals are similar in size but have very different organic coating layer structures: a compact ligand layer coats the Si nanocrystals; whereas, the Au nanocrystals are surrounded by a disordered ligand shell. The difference in capping ligand structure can be attributed to the significantly higher surface curvature of the significantly smaller Au nanocrystals. Additional simulations performed on flat $\mathrm{Au}$ and $\mathrm{Si}$ surfaces (nanocrystals of infinite radius, zero curvature) showed that the capping ligands formed a compact, solid-like organic layer in both cases (see Supporting Information). These results suggest the existence of a critical size for the particles, below which the nanoscale curvature effects appear.

The difference in experimentally observed interactions between the quatsomes and the Si and $\mathrm{Au}$ nanocrystals appears to result from the qualitatively different structure of the ligand shells on the nanocrystals. Figure 13 b,c illustrates a proposed mechanism for the interactions between nanocrystals and quatsomes. Both $\mathrm{Au}$ and $\mathrm{Si}$ nanocrystals aggregate in water due to their hydrophobic coating. As the nanocrystal aggregates or clusters form, they interact with other clusters of particles and with the hydrophilic surfaces of quatsomes. Let us consider first the case of $\mathrm{Au}$ particles (Figure 13b). The clusters of $\mathrm{Au}$ particles experience a repulsive interaction with the quatsome vesicles due to the hairy organic layer of the Au particles. This is 
due to the fact that the molecules of the hairy layer have less possible configurations near a surface (in this case, the surface of the quatsomes) than far from the surface, inducing a repulsion of entropic origin. This kind of entropic repulsive interaction plays a substantial role in selfassembly processes of nanoparticles. ${ }^{39}$ As a result, the Au particles aggregate into large clusters, without incorporation into the quatsomes. In the case of Si particles (Figure 13c), the entropic repulsive interaction is absent because the capping layer is compact in this case. Instead, we think that the interaction of a quatsome vesicle with a cluster of Si particles is reminiscent of the interaction of a vesicle with a hydrophobic solid surface. In this case, the layers of the quatsome vesicles were spread onto the surface of the clusters of Si particles. This spreading process requires first the breaking of vesicles and the input of energy. Vesicles are dynamic structures that can be reformed or reduced in size with the addition of sonication energy ${ }^{40-41}$ We found that when chol-CTAB quatsomes with SiNCs were sonicated for 15 or 30 min, they were smaller than those sonicated for 1 min or 5 min (Figure S5). Thus, it can be hypothesized that when quatsomes are sonicated they are broken down through a redistribution of membrane molecules. When the sonication is performed in the presence of hydrophobic SiNCs with a "compact organic layer" coverage, this redistribution would allow small aggregates of SiNCs to become stabilized by the hydrophobic part of cholesterol-CTAB monolayers (Figure 13c). The resulting self-assembled structure (clusters of Si particles covered by a quatsome monolayer) is now stable in water and acquires the radius corresponding to the spontaneous curvature of the quatsome building blocks (the bimolecular entities made by the association between CTAB and cholesterol). ${ }^{22}$ The result of this process is the coexistence between quatsome vesicles and these self-assembled clusters of Si covered by CTAB and cholesterol with a diameter similar to that of quatsome vesicles. 
a

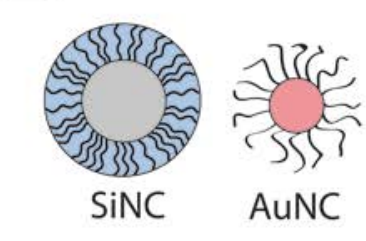

b
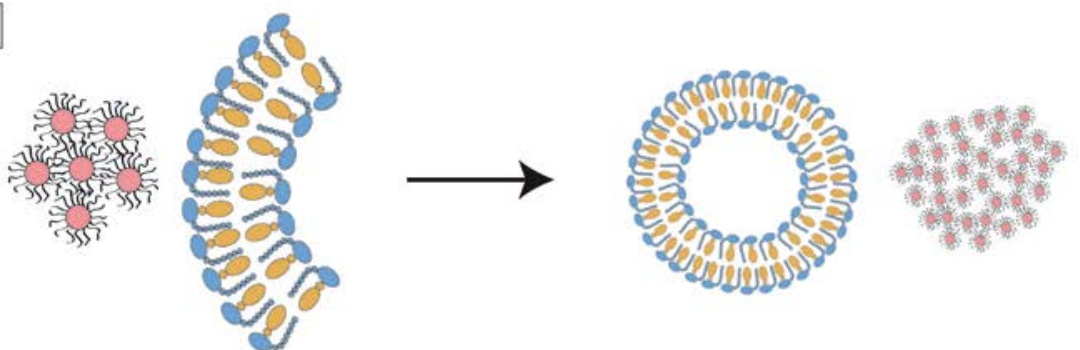

C
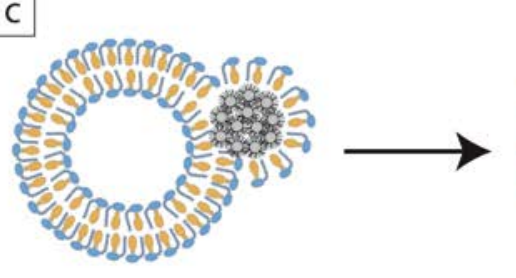
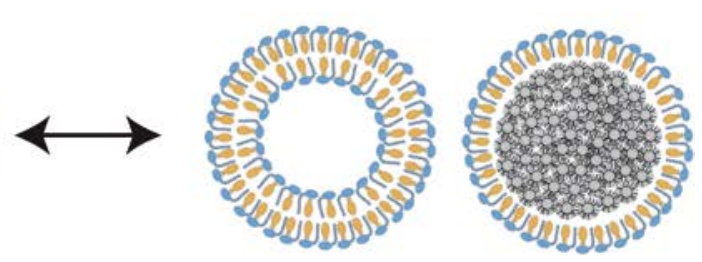

Figure 13. Proposed mechanism for the formation of quatsome-SiNC assemblies. In the diagram, CTAB is the blue headed structure, cholesterol is the yellow structure, AuNC are shown as red spheres and SiNc are shown as grey spheres. The ligands are shown schematically. (a) Scheme of the different structure of the organic layer covering SiNC and AuNC (compact versus hairy layer). (b) Scheme of the interactions of Au particles and quatsomes: the quatsomes do not form stable dispersions of AuNCs in water. (c) Scheme of the interactions of Si particles and quatsomes, and the formation of the stable Si nanocrystals covered with a monolayer of quatsomes

Although the nanoparticles are aggregated in quatsomes, it is still apparent that the synergy between cholesterol and CTAB, observed before in plain quatsomes, ${ }^{22}$ leads to the stable interaction between quatsomes and Si nanocrystals. When CTAB micelles were sonicated with Si nanocrystals, the Si nanocrystals appeared as either very small or very large, irregularly shaped clusters (Figure 8). Additionally, cryoTEM images of Si nanocrystals incorporated into quatsomes in Figure 2 show that the Si nanocrystal aggregations are approximately the same size as quatsomes without any SiNCs. Since the size of quatsomes is influenced by the interaction 
between cholesterol and CTAB, the size of Si nanocrystals aggregated suggests that they are associated with the same chol-CTAB units. Further, as shown in the dilution experiment in Figure 10, only Si nanocrystals dispersed in quatsomes remain stable after several rounds of dilution, while Si nanocrystals dispersed in CTAB micelles result in precipitation of the $\mathrm{Si}$ nanocrystals after multiple dilutions. Thus, it is specifically the quatsomes where cholesterol and CTAB interact as bimolecular building blocks that contribute to stabilizing the Si nanocrystals. We expect to be able to offer a more quantitative prediction in a near future, by combining our simulations with analytical expressions for interaction forces as in Ref 38.

\section{SUMMARY AND CONCLUSIONS}

A method to disperse fluorescent SiNCs in aqueous media with long-term stability was developed, utilizing chol-CTAB quatsomes, which are into non-liposomal vesicular structures. The stable SiNC aqueous dispersions can be made with five minutes of bath sonication. The NCs remain dispersed in water for several weeks and maintain the fluorescence properties of the SiNCs for several weeks and after dilution with additional water. The experimental data show that the association of SiNCs with chol-CTAB quatsomes is unique to the nanocrystal type (as compared to AuNCs) as well as the method of preparation. Quatsomes, which have been shown

to have extremely long term stability of at least three years in aqueous solutions, ${ }^{22}$ provide a vehicle for the dispersion of hydrophobic SiNCs into in-vitro or in-vivo environments, even under dilute conditions. The biocompatibility of quatsomes and near infrared emitting SiNCs make these structures excellent candidates for biomedical imaging applications. Furthermore, quatsomes have been shown to enhance protein activity and to protect proteins against premature degradation in topical pharmaceutical formulations, as well as to treat biofilms. ${ }^{26,27}$ Therefore, 
the incorporation of both biomolecules and SiNCs into quatsome structures offers the possibility to explore the behavior of these systems in biological environments and their use for theranostics.

\section{SUPPORTING INFORMATION}

Additional data is provided as supporting information: silicon nanocrystal characterization, calculation of number of SiNCs per quatsome, cryo-TEM images of SiNCs in $0.7 \mathrm{mM}$ cholesterol-CTAB quatsomes, cryo-TEM images of SiNCs in 7 mM CTAB, results of changing bath sonication time, results of using probe sonication or vortexing instead of bath sonication to incorporate SiNCs, results of changing volume ratio of SiNCs added to quatsomes, results of incorporating SiNCs into quatsomes with no ethanol in the water, incorporation of AuNCs into quatsomes, and details of molecular dynamics simulations. This material is available free of charge via the Internet at http://pubs.acs.org.

\section{ACKNOWLEDGEMENTS}

DAS and BAK acknowledge funding of this work by the Robert A. Welch Foundation (grant no. F-1464) and the US National Science Foundation (Grant no. CHE-1308813). This work was also supported by the DGI, Spain (Grant "Mother", MAT 2016-80826-R), by AGAUR, Generalitat de Catalunya (Grant 2014-SGR-17), the Networking Research Center on Bioengineering, Biomaterials, and Nanomedicine (CIBER-BBN) which is an initiative funded by the VI National R\&D\&I Plan 2008-2011, Iniciativa Ingenio 2010, Consolider Program, CIBER Actions, and by the Instituto de Salud Carlos III with assistance from the European Regional Development Fund. Also the Spanish Ministry of Economy and Competitiveness, through the 
"Severo Ochoa" Programme for Centres of Excellence in R \& D (SEV-2015-0496), is acknowledged. The ICTS "Nanbiosis", more specifically U6 Unit, is also acknowledged since some of the studies here reported have been performed there. LFT thanks the MICINN for her FPI bursary. JF acknowledges the CESGA Supercomputing center for technical support and computer time at the Finisterrae-II supercomputer.

\section{REFERENCES}

(1) Dubertret, B.; Skourides, P.; Norris, D. J.; Noireaux, V.; Brivanlou, A. H.; Libchaber, A. In Vivo Imaging of Quantum Dots Encapsulated in Phospholipid Micelles. Science 2002, 298, $1759-1762$.

(2) Michalet, X.; Pinaud, F. F.; Bentolila, L. A.; Tsay, J. M.; Doose, S.; Li, J. J.; Sundaresan, G.; Wu, A. M.; Gambhir, S. S.; Weiss, S. Quantum Dots for Live Cells, in Vivo Imaging, and Diagnostics. Science 2005, 307, 538-544.

(3) O'Farrell, N.; Houlton, A.; Horrocks, B. R. Silicon Nanoparticles: Applications in Cell Biology and Medicine. Int. J. Nanomedicine 2006, 1, 451-472.

(4) Panthani, M. G.; Khan, T. A.; Reid, D. K.; Hellebusch, D. J.; Rasch, M. R.; Maynard, J. A.; Korgel, B. A. In Vivo Whole Animal Fluorescence Imaging of a Microparticle-Based Oral Vaccine Containing $\left(\mathrm{CuInSe}_{\mathrm{x}} \mathrm{S}_{2-\mathrm{x}}\right) / \mathrm{ZnS}$ Core/Shell Quantum Dots. Nano Lett. 2013, 13, 4294-4298.

(5) Kovalenko, M. V.; Manna, L.; Cabot, A.; Hens, Z.; Talapin, D. V.; Kagan, C. R.; Klimov, V. I.; Rogach, A. L.; Reiss, P.; Milliron, D. J.; Guyot-Sionnest, P.; Konstantatos, G.; Park, 
W. J.; Hyeon, T.; Korgel, B. A.; Murray, C. B.; Heiss, W. Prospects of Nanoscience with Nanocrystals. ACS Nano 2015, 9, 1012-1057.

(6) Park, J.-H.; Gu, L.; Maltzahn, G. von; Ruoslahti, E.; Bhatia, S. N.; Sailor, M. J. Biodegradable Luminescent Porous Silicon Nanoparticles for in Vivo Applications. Nat. Mater. 2009, 8, 331-336.

(7) Henderson, E. J.; Shuhendler, A. J.; Prasad, P.; Baumann, V.; Maier-Flaig, F.; Faulkner, D. O.; Lemmer, U.; Wu, X. Y.; Ozin, G. A. Colloidally Stable Silicon Nanocrystals with NearInfrared Photoluminescence for Biological Fluorescence Imaging. Small 2011, 7, 25072516.

(8) Hessel, C. M.; Reid, D.; Panthani, M. G.; Rasch, M. R.; Goodfellow, B. W.; Wei, J.; Fujii, H.; Akhavan, V.; Korgel, B. A. Synthesis of Ligand-Stabilized Silicon Nanocrystals with Size-Dependent Photoluminescence Spanning Visible to Near-Infrared Wavelengths. Chem. Mater. 2012, 24, 393-401.

(9) Gu, L.; Hall, D. J.; Qin, Z.; Anglin, E.; Joo, J.; Mooney, D. J.; Howell, S. B.; Sailor, M. B. In Vivo Time-Gated Fluorescence Imaging with Biodegradable Luminescent Porous Silicon Nanoparticles. Nat. Commun. 2013, 4, 2236.

(10) Yu, Y.; Hessel, C. M.; Bogart, T. D.; Panthani, M. G.; Rasch, M. R.; Korgel, B. A. Room Temperature Hydrosilylation of Silicon Nanocrystals with Bifunctional Terminal Alkenes. Langmuir 2013, 29, 1533-1540.

(11) Erogbogbo, F.; Yong, K.-T.; Roy, I.; Xu, G.; Prasad, P. N.; Swihart, M. T. Biocompatible Luminescent Silicon Quantum Dots for Imaging of Cancer Cells. ACS Nano 2008, 2, 873878. 
(12) Jang, H.; Pell, L. E.; Korgel, B. A.; English, D. S. Photoluminescence Quenching of Silicon Nanoparticles in Phospholipid Vesicle Bilayers. J. Photochem. Photobiol. Chem. 2003, $158,111-117$.

(13) Paasonen, L.; Laaksonen, T.; Johans, C.; Yliperttula, M.; Kontturi, K.; Urtti, A. Gold Nanoparticles Enable Selective Light-Induced Contents Release from Liposomes. J. Controlled Release 2007, 122, 86-93.

(14) Rasch, M. R.; Rossinyol, E.; Hueso, J. L.; Goodfellow, B. W.; Arbiol, J.; Korgel, B. A. Hydrophobic Gold Nanoparticle Self-Assembly with Phosphatidylcholine Lipid: Membrane-Loaded and Janus Vesicles. Nano Lett. 2010, 10, 3733-3739.

(15) Hessel, C. M.; Rasch, M. R.; Hueso, J. L.; Goodfellow, B. W.; Akhavan, V. A.; Puvanakrishnan, P.; Tunnel, J. W.; Korgel, B. A. Alkyl Passivation and Amphiphilic Polymer Coating of Silicon Nanocrystals for Diagnostic Imaging. Small 2010, 6, 20262034.

(16) Erogbogbo, F.; Yong, K.-T.; Roy, I.; Hu, R.; Law, W.-C.; Zhao, W.; Ding, H.; Wu, F.; Kumar, R.; Swihart, M. T.; Prasad, P. N. In Vivo Targeted Cancer Imaging, Sentinel Lymph Node Mapping and Multi-Channel Imaging with Biocompatible Silicon Nanocrystals. ACS Nano 2010, 5, 413-423.

(17) Al-Jamal, W. T.; Kostarelos, K. Liposomes: From a Clinically Established Drug Delivery System to a Nanoparticle Platform for Theranostic Nanomedicine. Acc. Chem. Res. 2011, 44, 1094-1104.

(18) Rasch, M. R.; Yu, Y.; Bosoy, C.; Goodfellow, B. W.; Korgel, B. A. ChloroformEnhanced Incorporation of Hydrophobic Gold Nanocrystals into 
Dioleoylphosphatidylcholine (DOPC) Vesicle Membranes. Langmuir 2012, 28, 1297112981.

(19) Sawant, R. R.; Torchilin, V.P. Liposomes as 'Smart' Pharmaceutical Nanocarriers. Soft Matter 2010, 6, 4026-4044.

(20) European Medicines Agency: Scientific Guidelines: Nanomedicines. http://www.ema.europa.eu/ema/index.jsp?curl=pages/regulation/general/general_content_0 00564.jsp\&mid=WC0b01ac05806403e0 (Accessed Feb 6, 2016).

(21) U.S. Department of Health and Human Services, Food and Drug Administration, Center for Drug Evaluation and Research (CDER): Guidance for Industry: Liposome Drug Products (Draft $\quad$ Guidance, $\quad$ Revision $\quad 1, \quad$ October 2015). http://www.fda.gov/downloads/drugs/guidancecomplianceregulatoryinformation/guidances/ucm 070570.pdf (Accessed Sept 18, 2017).

(22) Ferrer-Tasies, L.; Moreno-Calvo, E.; Cano-Sarabia, M.; Aguilella-Arzo, M.; Angelova, A.; Lesieur, S.; Ricart, S.; Faraudo, J.; Ventosa, N.; Veciana, J. Quatsomes: Vesicles Formed by Self-Assembly of Sterols and Quaternary Ammonium Surfactants. Langmuir 2013, 29, 6519-6528.

(23) Elizondo, E.; Larsen, J.; Hatzakis, N. S.; Cabrera, I.; Bjørnholm, T.; Veciana, J.; Stamou, D.; Ventosa, N. Influence of the Preparation Route on the Supramolecular Organization of Lipids in a Vesicular System. J. Am. Chem. Soc. 2012, 134, 1918-1921.

(24) Cabrera, I.; Elizondo, E.; Esteban, O.; Corchero, J. L.; Melgarejo, M.; Pulido, D.; Córdoba, A.; Moreno, E.; Unzueta, U.; Vazquez, E.; Abasolo, I.; Schwartz, S.; Villaverde, A.; Albericio, F.; Royo, M.; Garcia-Parajo, M. F.; Ventosa, N.; Veciana, J. Multifunctional 
Nanovesicle-Bioactive Conjugates Prepared by a One-Step Scalable Method Using CO2Expanded Solvents. Nano Lett. 2013, 13, 3766-3774.

(25) Grimaldi, N.; Andrade, F.; Segovia, N.; Ferrer-Tasies, L.; Sala, S.; Veciana, J.; Ventosa, N. Lipid-based Nanovesicles for Nanomedicine. Chem. Soc. Rev. 2016, 45, 6520-6545.

(26) Ventosa, N.; Cabrera, I.; Veciana, J.; Santana, H.; Martinez, E.; Berlanga, J. A. Vesicles Comprising Epidermal Growth Factor and Compositions That Contain Them. CU20120112, 2012.

(27) Thomas, N.; Dong, D.; Richter, K.; Ramezanpour, M.; Vreugde, S.; Thierry, B.; Wormald, P.-J.; Prestidge, C. A. Quatsomes for the Treatment of Staphylococcus Aureus Biofilm. J. Mater. Chem. B 2015, 3, 2770-2777.

(28) Liu, X.; Ardizonne, A.; Sui, B.; Anzola, M.; Ventosa, N.; Liu, T.; Veciana, J.; Belfield, K.D. Fluorenyl-Loaded Quatsome Nanostructured Fluorescent Probes. ACS Omega, 2017, 2, 4112-4122.

(29) U.S. Department of Health and Human Services, Food and Drug Administration, Inactive $\begin{array}{lllll}\text { Ingredient } & \text { Search } & \text { for } & \text { Approved }\end{array}$ https://www.accessdata.fda.gov/scripts/cder/iig/index.cfm (Accessed Nov 25, 2017).

(30) Phillips, J. C.; Braun, R.; Wang, W.; Gumbart, J.; Tajkhorshid, E.; Villa, E.; Chipot, C.; Skeel, R. D.; Kalé, L.; Schulten, K. Scalable Molecular Dynamics with NAMD. J. Comput. Chem. 2005, 26, 1781-1802.

(31) Vanommeslaeghe, K.; Hatcher, E.; Acharya, C.; Kundu, S.; Zhong, S.; Shim, J.; Darian, E.; Guvench, O.; Lopes, P.; Vorobyov, I.; Mackarell, A. D. CHARMM General Force Field: A Force Field for Drug-like Molecules Compatible with the CHARMM All-Atom Additive Biological Force Fields. J. Comput. Chem. 2010, 31, 671-690. 
(32) Marques, E. F. Size and stability of catanionic vesicles: effects of formation path, sonication, and aging. Langmuir 2000, 16, 4798-4807.

(33) Alenaizi, R.; Radiman, S.; Abdul Rahman, I.; Mohamed, F. Zwitterionic BetaineCholesterol System: Effects of Sonication Duration and Aging on Vesicles Stability. Colloid Surf., A 2015, 482, 662-669.

(34) Antonietti, M.; Förster, S. Vesicles and Liposomes: A Self-Assembly Principle Beyond Lipids. Adv. Mater. 2003, 15, 1323-1333.

(35) Gao, J.; Bender, C. M.; Murphy, C. J. Dependence of the Gold Nanorod Aspect Ratio on the Nature of the Directing Surfactant in Aqueous Solution. Langmuir 2003, 19, 90659070.

(36) Sau, T. K.; Murphy, C. J. Room Temperature, High-Yield Synthesis of Multiple Shapes of Gold Nanoparticles in Aqueous Solution. J. Am. Chem. Soc. 2004, 126, 8648-8649.

(37) Smith, D. K.; Korgel, B. A. The Importance of the CTAB Surfactant on the Colloidal SeedMediated Synthesis of Gold Nanorods. Langmuir 2008, 24, 644-649.

(38) Humphrey, W.; Dalke, A.; Schulten, K. VMD: Visual Molecular Dynamics. J. Mol. Graphics 1996, 14, 33-38.

(39) Sánchez-Iglesias, A.; Grzelczak, M.; Altantzis, T.; Goris, B.; Pérez-Juste, J.; Bals, S.; Van Tendeloo, G.; Donaldson, S. H.; Chmelka, B. F.; Israelachvili, J. N.; Liz-Marzan, L. M. Hydrophobic Interactions Modulate Self-Assembly of Nanoparticles. ACS Nano 2012, 6, 11059-11065.

(40) Marsh, D. Handbook of Lipid Bilayers, Second Edition; CRC Press, 2013.

(41) Szoka, F.; Papahadjopoulos, D. Comparative Properties and Methods of Preparation of Lipid Vesicles (Liposomes). Annu. Rev. Biophys. Bioeng. 1980, 9, 467-508. 
TABLE OF CONTENTS FIGURE

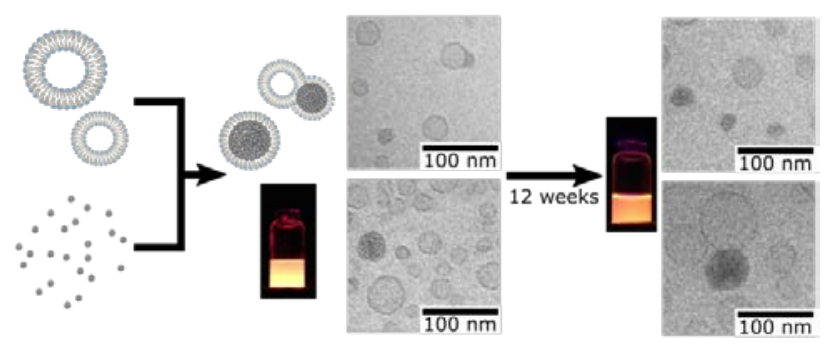

